\title{
Growth and Collapse of a Bose-Einstein Condensate with Attractive Interactions
}

\author{
C. A. Sackett, ${ }^{1}$ H. T. C. Stoof, ${ }^{1,2}$ and R. G. Hulet ${ }^{1}$ \\ ${ }^{1}$ Physics Department and Rice Quantum Institute, Rice University, Houston, Texas 77251-1892 \\ ${ }^{2}$ Institute for Theoretical Physics, University of Utrecht, Princetonplein 5, 3584 CC Utrecht, The Netherlands
}

(Received 23 October 1997)

\begin{abstract}
We consider the dynamics of a quantum degenerate trapped gas of ${ }^{7} \mathrm{Li}$ atoms. Because the atoms have a negative $s$-wave scattering length, a Bose condensate of ${ }^{7} \mathrm{Li}$ becomes mechanically unstable when the number of condensate atoms approaches a maximum value. We calculate the dynamics of the collapse that occurs when the unstable point is reached. In addition, we use the quantum Boltzmann equation to investigate the nonequilibrium kinetics of the atomic distribution during and after evaporative cooling. The condensate is found to undergo many cycles of growth and collapse before a stationary state is reached. [S0031-9007(98)05489-1]

PACS numbers: 03.75.Fi, 32.80.Pj, 42.50.Vk, 67.40.-w
\end{abstract}

The recent observations of Bose-Einstein condensation (BEC) in weakly interacting gases [1] has enabled a series of beautiful experiments that probe the dynamics of the condensate. The frequency and damping of the collective modes of a condensate [2], propagation of sound in a condensate [3], and, recently, the growth of the condensate [4] have been reported. Although these experiments have used atoms with positive $s$-wave scattering lengths, we show in this paper that the dynamical behavior of a negative scattering length gas, such as ${ }^{7} \mathrm{Li}$, is especially interesting, and offers the opportunity to directly observe and study macroscopic quantum tunneling.

A negative scattering length $a$ implies effectively attractive interactions. In a spatially homogeneous gas, these interactions lead to ordinary classical condensation into a liquid or solid, preventing Bose-Einstein condensation in the metastable region of the phase diagram [5]. However, confinement in an atom trap produces stabilizing forces that enable the formation of a metastable BoseEinstein condensate, if the number of condensed atoms is less than some maximum number $N_{m}$. For a harmonic trap, Ruprecht et al. [6] showed that in mean-field theory $N_{m} \simeq 0.57 l /|a|$, where $l=(\hbar / m \omega)^{1 / 2}$ is the extent of the one-particle ground state in the harmonic trap [7]. For the ${ }^{7} \mathrm{Li}$ experiments of Ref. [8], $N_{m} \simeq 1400$ atoms, which agrees with the measured value.

Although a condensate can exist in a trapped gas, it is predicted to be metastable and to decay by quantum or thermal fluctuations [9-11]. The condensate has only one unstable collective mode, which in the case of an isotropic trap corresponds to the breathing mode $[12,13]$. The condensate therefore collapses as a whole, either by thermal excitation over or by quantum mechanical tunneling through a macroscopic energy barrier in configuration space. The probability of forming small, dense clusters is greatly suppressed because of the large energy barrier for this process, compared to that for the breathing mode. This suppression can also be understood from the fact that the typical length scale for fluctua- tions of the condensate is the healing length, which is approximately equal to the condensate size near the instability point.

Experimentally, it is also important to understand how such a condensate can be formed from a noncondensed cloud by means of evaporative cooling. This question was recently addressed by Gardiner et al. in the context of experiments with gases having $a>0$ [14]. These authors neglect the coherent dynamics of the condensate and focus instead on the kinetics of condensation [15]. By treating the noncondensed atoms as a static "heat bath," they are able to derive a simple equation for the growth of the number of condensate particles that appears to fit well with experimental results [4]. The same approach, however, does not work in the case of atomic ${ }^{7} \mathrm{Li}$ because it does not allow for the collapse of the condensate. Moreover, the process of evaporative cooling leads to dynamical changes in the noncondensed heat bath. The study of these effects on the dynamics of the condensate is the main topic of this Letter. Some preliminary results have already appeared in a recent review article [16].

When collisions can be neglected, the dynamics of the condensate wave function $\psi$ for a gas with $a<0$ is well described by the nonlinear Schrödinger (or GrossPitaevskii [17]) equation

$$
\begin{aligned}
i \hbar \frac{\partial \psi(\mathbf{x}, t)}{\partial t}= & \left(-\frac{\hbar^{2} \nabla^{2}}{2 m}+V(\mathbf{x})\right) \psi(\mathbf{x}, t) \\
& +T^{2 B}(\mathbf{0}, \mathbf{0} ; 0)|\psi(\mathbf{x}, t)|^{2} \psi(\mathbf{x}, t) .
\end{aligned}
$$

Here $m$ denotes the mass of ${ }^{7} \mathrm{Li}, T^{2 B}(\mathbf{0}, \mathbf{0} ; 0)=4 \pi a \hbar^{2} / m$ is the two-body $T$ matrix, and $a$ is the scattering length of $-27 a_{0}$ [18]. For the external trapping potential $V(\mathbf{x})$ we take a harmonic potential with an effective isotropic level splitting $\hbar \omega=\hbar\left(\omega_{x} \omega_{y} \omega_{z}\right)^{1 / 3}$ of $7 \mathrm{nK}$ [8]. Note that we ignore the mean-field contribution from the noncondensed atoms [19], because it is nearly constant over the size of the condensate and therefore only slightly affects the condensate dynamics. 
This description is semiclassical, and also neglects quantum and thermal fluctuations. These fluctuations are most easily incorporated by means of the partition function of the condensate, which is a functional integral $\int d\left[\psi^{*}\right] d[\psi] \exp \left(-S\left[\psi^{*}, \psi\right] / \hbar\right)$ over all periodic configurations of the condensate, with a weight determined by the (Euclidean) action of the nonlinear Schrödinger equation. This partition function is calculated in terms of the density and phase of the condensate, defined by $\psi=\sqrt{\rho} e^{i \chi}$. The Gaussian integral over the phase field $\chi$ can be performed exactly, leaving only the determination of the functional integral $\int d[\rho] \exp (-S[\rho] / \hbar)$.

Unfortunately, this integral cannot be calculated exactly. However, since we are primarily interested in the dynamics of the unstable breathing mode of the condensate, we can use a variational method $[11,20]$ and consider only Gaussian density profiles

$$
\rho(\mathbf{x} ; q(t))=N_{0}\left(\frac{1}{\pi q^{2}(t)}\right)^{3 / 2} \exp \left(-\frac{\mathbf{x}^{2}}{q^{2}(t)}\right) .
$$

Substituting this profile in the action $S[\rho]$, we find that the dynamics of the condensate is equivalent to the dynamics of a fictitious particle with mass $m^{*}=3 N_{0} m / 2$ in the unstable potential [21]

$$
U(q)=N_{0}\left(\frac{3 \hbar^{2}}{4 m q^{2}}+\frac{3}{4} m \omega^{2} q^{2}-\frac{N_{0}}{\sqrt{2 \pi}} \frac{\hbar^{2}|a|}{m q^{3}}\right) .
$$

The rate of decay for both quantum tunneling and thermal fluctuations can be calculated within this formalism [11] and are shown in Fig. 1. For large numbers of condensate atoms, these collective decay mechanisms are much faster than the decay caused by inelastic two- and three-body collisions, since the energy barrier out of the metastable minimum vanishes as $N_{0}$ approaches $N_{m}$. At experimentally relevant temperatures of 300 to $500 \mathrm{nK}$, the

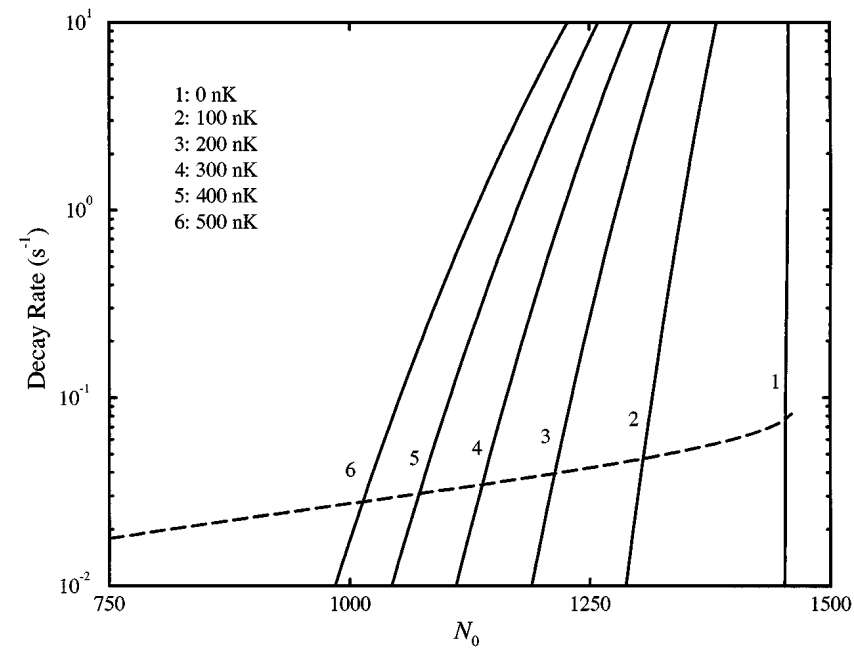

FIG. 1. Decay rate of the condensate as a function of the number of condensate particles at 0,100, 200,300, 400, and $500 \mathrm{nK}$. The dashed line shows the decay due to inelastic two and three-body collisions [22]. collective decay processes dominate for $N_{0}$ greater than about 1100 atoms. Besides the calculation of the decay rates, the above analogy also allows study of the dynamics of the collapse that occurs after the condensate has been driven out of its metastable minimum. A typical example is shown in Fig. 2. At first, the condensate collapses with increasing speed along the potential hill outside the barrier. However, during the collapse, the condensate density grows rapidly, thereby increasing the decay rate from inelastic two- and three-body collisions. Atoms that inelastically collide acquire substantial energy and are ejected from the trap. Because of these loss mechanisms, the collapse is arrested when the number of condensate atoms is of the order of one. Atoms are lost so quickly that the density of the condensate always obeys $n|a|^{3} \ll 1$, which is a necessary requirement for the validity of the nonlinear Schrödinger equation (1). If there were no inelastic collisions, the condensate would fully collapse [23], and a more complex theory would be needed. In principle, fluctuations still become important at the end of the collapse when $N_{0}$ is about 1 , but this should hardly affect Fig. 2.

The above remarks pertain to the dynamics of the condensate in the absence of collisions with noncondensed atoms, but so far all experiments producing BEC have relied on evaporative cooling, which requires such collisions. Therefore, we investigate the kinetics of condensation in a trapped gas, using the Boltzmann transport equation in a way similar to the treatment of evaporative cooling in Refs. [24,25]. In this approach, the semiclassical distribution function $f(\mathbf{x}, \mathbf{p})$ is defined such that the number of atoms at position $\mathbf{x}$ with momentum $\mathbf{p}$ is $d \mathbf{x} d \mathbf{p} f(\mathbf{x}, \mathbf{p}) /(2 \pi \hbar)^{3}$. The evolution of $f$ is given by [26]

$$
\frac{d}{d t} f(\mathbf{x}, \mathbf{p}, t)=I(\mathbf{x}, \mathbf{p})-\Gamma(\mathbf{x}) f(\mathbf{x}, \mathbf{p}),
$$

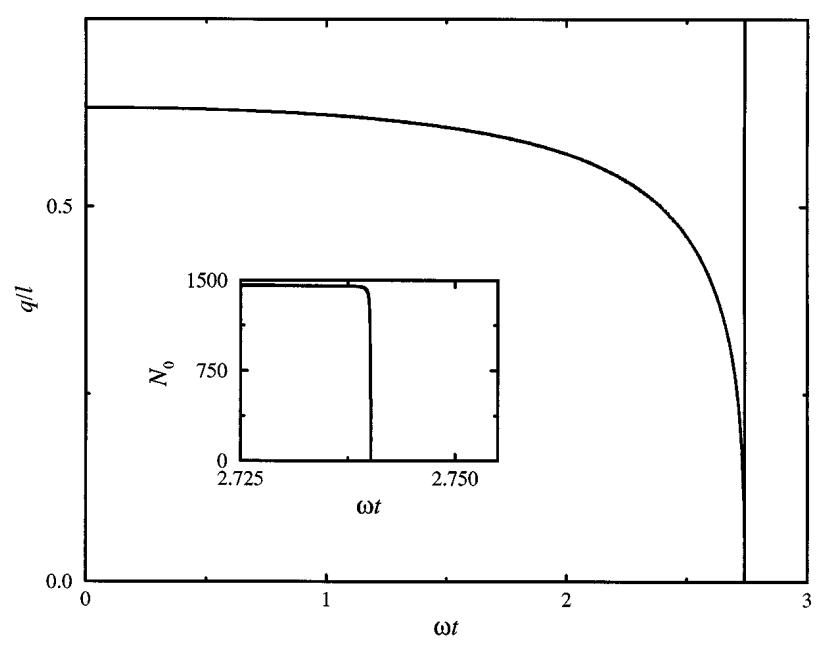

FIG. 2. Typical evolution of the condensate during collapse. The main figure shows the size of the condensate, and the inset shows the number of condensate atoms. 
where the derivative on the left is the total time derivative. The effect of elastic collisions is expressed as

$$
\begin{aligned}
I\left(\mathbf{x}, \mathbf{p}_{1}\right)= & \frac{\sigma}{4 \pi^{4} m \hbar^{3}} \int d \mathbf{p}_{2} d \mathbf{p}_{3} d \mathbf{p}_{4} \delta^{3}\left(\mathbf{p}_{1}+\mathbf{p}_{2}-\mathbf{p}_{3}-\mathbf{p}_{4}\right) \delta\left(p_{1}^{2}+p_{2}^{2}-p_{3}^{2}-p_{4}^{2}\right) \\
& \times\left[f_{3} f_{4}\left(1+f_{1}\right)\left(1+f_{2}\right)-f_{1} f_{2}\left(1+f_{3}\right)\left(1+f_{4}\right)\right],
\end{aligned}
$$

where the factors $\left(1+f_{i}\right)$ account for the Bose statistics of the atoms. Here $f_{i}$ stands for $f\left(\mathbf{x}, \mathbf{p}_{i}\right)$ and $\sigma=8 \pi a^{2}$. The loss rate from the trap is given by $\Gamma(\mathbf{x})=G_{1}+$ $G_{2} \int d \mathbf{p} f(\mathbf{x}, \mathbf{p}) /(2 \pi \hbar)^{3}$, where the terms with $G_{1}$ and $G_{2}$ reflect, respectively, collisions with hot background gas atoms and inelastic collisions between trapped atoms.

As for the case of a classical gas [24,25], Eq. (4) can be simplified by taking the motion of the atoms to be ergodic, so that an atom with a given energy will sample each available element of $\{\mathbf{x}, \mathbf{p}\}$ space with equal probability. The distribution function $f(\mathbf{x}, \mathbf{p})$ can then be expressed in terms of the energy distribution function $f(E)$, through the relation

$$
f(\mathbf{x}, \mathbf{p})=\int d E \delta(H(\mathbf{x}, \mathbf{p})-E) f(E),
$$

where $H(\mathbf{x}, \mathbf{p})=p^{2} / 2 m+V(\mathbf{x})$ is the classical Hamiltonian. The differential equation for $f(E, t)$ is derived by substituting (6) into (4), as is described in detail in Refs. [24,25]. The only difference here is the use of Bose statistics, which requires the insertion of factors $1+f\left(E_{i}\right)$ as in Eq. (5). Although the semiclassical approximation results in a continuous function $f(E)$, the quantum nature of the trapped gas can be recovered by restricting $f(E)$ to values $E_{n}=(n+3 / 2) \hbar \omega$. Note that $f(E, t)$ can also be determined using a Monte Carlo technique [27].

The above model neglects the effect of the mean-field interaction energy. Per atom, however, this interaction energy is limited to about $\hbar \omega$ by the stability criterion arising from $a$ being negative, which limits the effects of the mean-field energy on the kinetics of the gas. The accuracy of the approximations can be checked by considering collisions between condensate atoms, since the mean-field interaction is largest in the condensate and the semiclassical approximation is least accurate for the ground state of the trap. We compare the rates for inelastic collisions between condensate atoms, $G_{2} \int d \mathbf{x} \rho(\mathbf{x})^{2}$, which depend only on the density distribution. The semiclassical distribution derived from Eq. (6) is

$$
\rho(\mathbf{x})=\left(\frac{2}{3 \pi}\right)^{2}\left(\frac{N_{0}}{l^{3}}\right)\left(3-\frac{x^{2}}{l^{2}}\right)^{1 / 2},
$$

while the exact distribution is given by Eq. (2). From these distributions, the ratio of the exact and approximate collision rates is $1.2(l / q)^{3}$. When interactions are small, then $q \simeq l$, and the error caused by the semiclassical approximation is only $20 \%$. However, the mean-field interaction causes $q$ to decrease as $N_{0}$ grows. The variational cal- culations gives $q=0.67 l$ just before the collapse. The collision rate is then inaccurate by a factor of 4 , and collision terms for other low-lying states will have similar, though smaller, errors. Although these errors are significant, they only occur for $N_{0}$ relatively close to $N_{m}$. For instance, at $N_{0} \simeq 0.8 N_{m}$, the error is a factor of 2 . Because $N_{0} \geq 0.8 N_{m}$ for only a small fraction of time, the approximations are still useful, but for large $N_{0}$ the quantitative predictions of the model will be somewhat inaccurate.

The possibility for the condensate to collapse is included in the model using the decay rates given in Fig. 1. A random number is chosen to determine whether a decay occurs during an integration time step, and when one does the condensate number is set to zero reflecting the rapid loss shown in Fig. 2. Also, evaporative cooling is included by setting $f(E)=0$ for $E>E_{c}(t)$, where $E_{c}(t)$ is the experimentally set cutoff energy.

The response of the condensate number to evaporative cooling is shown in Fig. 3. The condensate alternately fills and collapses, until finally the phase-space density of the gas is too low to reach $N_{0} \simeq N_{m}$. The slow final decay is due to the trap losses. Qualitatively similar behavior has been found after submission of this Letter by Kagan et al. [28], although we differ quantitatively in the number of condensate atoms remaining after a collapse.

In Fig. 3, evaporative cooling was halted at $t=5 \mathrm{~s}$, but the oscillations in $N_{0}$ persist for an anomalously long time compared to the elastic collision time $\tau_{c}=1 /\langle n \sigma v\rangle \simeq$ $0.8 \mathrm{~s}$. In order to investigate this phenomenon further, the

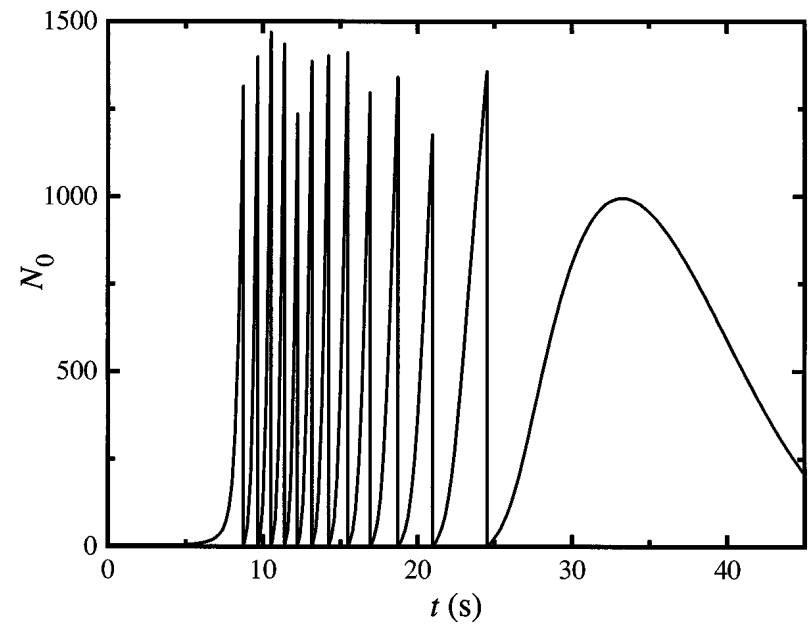

FIG. 3. Typical evolution of condensate number $N_{0}$ in response to evaporative cooling. The gas initially consists of $4 \times 10^{5}$ atoms at $500 \mathrm{nK}$. During the first $5 \mathrm{~s}$ of evolution, roughly $40 \%$ of the atoms are removed by evaporative cooling, after which evaporation is halted. 


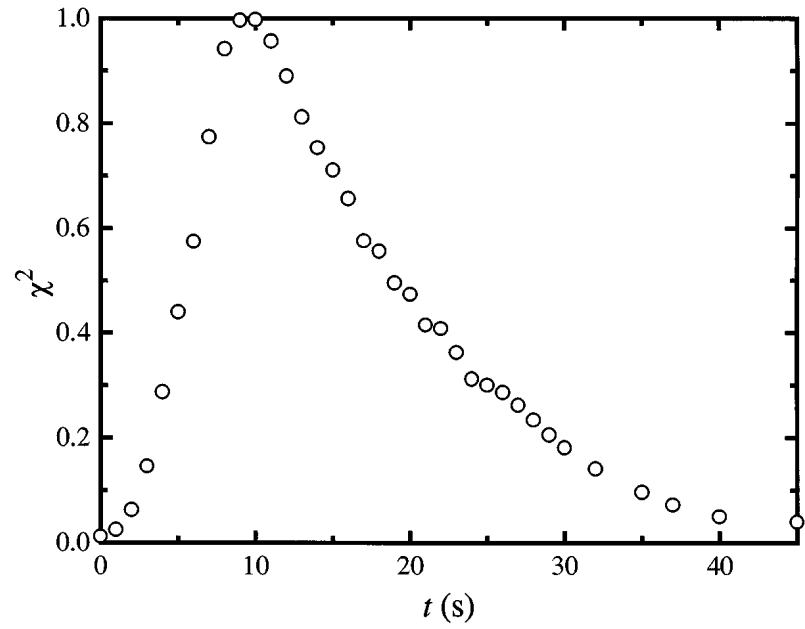

FIG. 4. Equilibration of a degenerate gas. Using the same conditions as in Fig. 3, at each time the density distribution is fit to an equilibrium density distribution, and the resulting value of $\chi^{2}$ plotted.

density profiles generated by the model were fit to equilibrium Bose-Einstein distributions at various times. The values of $\chi^{2}$ resulting from the fits are plotted in Fig. 4. After reaching degeneracy, the curve shows an approximately exponential approach to equilibrium, with a time constant of about $10 \tau_{c}$. The same test performed on a nondegenerate cloud initially prepared in a nonequilibrium state yields an equilibration time of $5 \tau_{c}$. The slower approach to equilibrium for the degenerate case is presumably caused by the small phase-space volume of the condensate, and the fact that due to the limit $N_{0}<N_{m}$, the stimulated Bose scattering factors in Eq. (4) cannot become as enormous as they might in the $a>0$ case. Furthermore, the oscillations in $N_{0}$ will persist until the distribution is very close to equilibrium, since the total number of atoms in the trap is much greater than $N_{m}$.

Note, finally, that the evolution in Fig. 3 represents only one possible outcome of evaporative cooling, and that because of the stochastic nature of the collapse a given evolution is not repeatable. Experimental measurements of $N_{0}$ in the degenerate regime should, however, exhibit large fluctuations between 0 and $N_{m}$. Observation of such fluctuations and measurement of their statistics would provide confirmation of the collective nature of the collapse. If sufficiently low temperatures can be reached, these fluctuations would be evidence of macroscopic quantum tunneling. We therefore believe that a dilute Bose gas with $a<0$ presents unique opportunities for studying the dynamical properties of a condensate.

[1] M.J. Anderson et al., Science 269, 198 (1995); C.C. Bradley et al., Phys. Rev. Lett. 75, 1687 (1995); K. B.
Davis et al., Phys. Rev. Lett. 75, 3969 (1995).

[2] D. S. Jin et al., Phys. Rev. Lett. 77, 420 (1996); M.-O. Mewes et al., Phys. Rev. Lett. 77, 988 (1996); D. S. Jin et al., Phys. Rev. Lett. 78, 764 (1997).

[3] M. R. Andrews et al., Phys. Rev. Lett. 79, 553 (1997).

[4] W. Ketterle (private communication).

[5] For the temperature $T=0$ case see L.D. Landau and E. M. Lifshitz, Statistical Physics (Pergamon, London, 1958). The case $T>0$ is discussed in H.T.C. Stoof, Phys. Rev. A 49, 3824 (1994).

[6] P. A. Ruprecht et al., Phys. Rev. A 51, 4704 (1995).

[7] This is the $T=0$ result. For $T>0$, the number is somewhat reduced. See for more details M. Houbiers and H. T. C. Stoof, Phys. Rev. A 54, 5055 (1996).

[8] C. C. Bradley, C. A. Sackett, and R. G. Hulet, Phys. Rev. Lett. 78, 985 (1997).

[9] Yu. Kagan, G. V. Shlyapnikov, and J.T.M. Walraven, Phys. Rev. Lett. 76, 2670 (1996).

[10] E. V. Shuryak, Phys. Rev. A 54, 3151 (1996).

[11] H. T. C. Stoof, J. Stat. Phys. 87, 1353 (1997).

[12] R. J. Dodd et al., Phys. Rev. A 54, 661 (1996).

[13] K. G. Singh and D. S. Rokhsar, Phys. Rev. Lett. 77, 1667 (1996).

[14] C. W. Gardiner et al., Phys. Rev. Lett. 79, 1793 (1997).

[15] H. T. C. Stoof, Phys. Rev. Lett. 78, 768 (1997).

[16] C. A. Sackett et al., Appl. Phys. B 65, 433 (1997).

[17] L.P. Pitaevskii, Sov. Phys. JETP 13, 451 (1961); E.P. Gross, J. Math. Phys. (N.Y.) 4, 195 (1963).

[18] E. R. I. Abraham et al., Phys. Rev. Lett. 74, 1315 (1995).

[19] V. V. Goldman, I. F. Silvera, and A. J. Leggett, Phys. Rev. B 24, 2870 (1981).

[20] V.M. Pérez-Garciá et al., Phys. Rev. Lett. 77, 5320 (1996).

[21] If the density field is expanded as

$$
\rho(\mathbf{x} ; q(t))+\sum_{n l m}{ }^{\prime} C_{n l m}(t) e^{-\mathbf{x}^{2} / 2 q^{2}(t)} \phi_{n l m}(\mathbf{x} ; q(t)),
$$

where $\phi_{n l m}(\mathbf{x} ; q)$ are the usual harmonic oscillator states with frequency $\omega_{q}=\hbar / m q^{2}$, and the sum does not include the states $\phi_{000}$ and $\phi_{100}$, we can systematically improve upon this result by expanding the action $S[\rho]$ up to quadratic order in $C_{n l m}$ and integrating out the coefficients describing the dynamics of all the stable collective modes.

[22] A. J. Moerdijk and B.J. Verhaar, Phys. Rev. A 53, R19 (1996).

[23] See also L. P. Pitaevskii, Phys. Lett. A 221, 14 (1996).

[24] O. J. Luiten, M.W. Reynolds, and J.T.M. Walraven, Phys. Rev. A 53, 381 (1996).

[25] C. A. Sackett, C. C. Bradley, and R. G. Hulet, Phys. Rev. A 55, 3797 (1997).

[26] See, for instance, K. Huang, Statistical Mechanics (John Wiley and Sons, New York, 1987).

[27] M. Holland, J. Williams, and J. Cooper, Phys. Rev. A 55, 3670 (1997).

[28] Yu. Kagan, A.E. Muryshev, and G. V. Shlyapnikov (unpublished). 